\title{
One-armed oscillations in Be star discs
}

\author{
J. C. B. Papaloizou ${ }^{1}$ and G. J. Savonije ${ }^{2}$ \\ 1 Department of Applied Mathematics and Theoretical Physics, Centre for Mathematical Sciences, Wilberforce Road, \\ Cambridge CB3 0WA, UK \\ 2 Astronomical Institute "Anton Pannekoek", University of Amsterdam, Kruislaan 403, 1098 SJ Amsterdam, The Netherlands \\ e-mail: gertjan@science.uva.nl
}

Received 11 April 2006 / Accepted 20 June 2006

ABSTRACT

\begin{abstract}
Aims. In this paper we study the effect of the quadrupole-term in the gravitational potential of a rotationally deformed central Be star on one armed density waves in the circumstellar disc. The aim is to explain the observed long-term violet over red (V/R) intensity variations of the double peaked Balmer emission-lines, not only in cool Be star systems, but also in the hot systems like $\gamma$ Cas.

Methods. We have carried out semi-analytic and numerical studies of low-frequency one armed global oscillations in near Keplerian discs around Be stars. In these we have investigated surface density profiles for the circumstellar disc which have inner narrow low surface density or gap regions, just interior to global maxima close to the rapidly rotating star, as well as the mode inner boundary conditions.

Results. Our results indicate that it is not necessary to invoke extra forces such as caused by line absorption from the stellar flux in order to explain the long-term V/R variations in the discs around massive Be stars. When there exists a narrow gap between the star and its circumstellar disc, with the result that the radial velocity perturbation is non-zero at the inner disc boundary, we find oscillation (and $\mathrm{V} / \mathrm{R}$ ) periods in the observed range for plausible magnitudes for the rotational quadrupole term.
\end{abstract}

Key words. stars: emission-line, Be

\section{Introduction}

Many Be stars exhibit long-term periodicities in the relative intensity of the double peaked Balmer emission lines, the so-called $\mathrm{V} / \mathrm{R}$ variations, which occur on timescales of typically a few years to a few decades, see e.g. Dachs (1987), Hubert (1994) and Okazaki (1997) and references therein. Okazaki (1991) has suggested that a one armed $(m=1)$ oscillation mode in the circumstellar disc about the Be star is responsible for the V/R variations. In a near Keplerian disc a one-armed mode shows up as a very slowly revolving perturbation pattern: the locally enhanced temperature and density causes stronger emission in one part of the disc. This results in either the violet- or red component of the double Balmer emission peaks to be brighter than normal when the compressed enhanced emission region is located in the approaching, respectively receding part of the disc. Okazaki (1991) applied a point mass potential for the Be star and obtained a slowly precessing global $m=1$ pattern by the invoked pressure perturbations. However, in this way one generates retrograde modes which tend to propagate throughout the disc, while observations showed the $\mathrm{V} / \mathrm{R}$ variations to occur only near the central star. Papaloizou et al. (1992) improved on this by considering a natural extension of this model by including the quadrupole-term in the gravitational potential of the rapidly rotating and thus flattened Be star. Deviation from a $1 / r$ point mass potential is known to give rise to precessing elliptic particle orbits. In a gaseous disc the deviation from a point mass potential leads naturally to slowly revolving prograde $m=1 \mathrm{os}-$ cillation modes (if excited) which are confined to a region within a few stellar radii from the star. Papaloizou et al. (1992) showed that the propagation region extends in fact from the inner disc boundary up to about the point where the modal pattern speed equals the local particle precession speed. The radial size of this natural oscillation cavity is of order the stellar radius $R_{\mathrm{s}}$ as the quadrupole component decays rapidly with increasing distance from the star.

Meanwhile observations have shown that the perturbation pattern in several Be star-disc systems with $\mathrm{V} / \mathrm{R}$ variations revolves indeed in a prograde sense, e.g. in $\beta^{1}$ Mon (Telting et al. 1994). Hummel \& Vrancken (1997) and Hummel \& Hanuschik (1997) calculated line profiles from discs with revolving $m=1$ modal patterns and showed that these eccentric modes can explain the observed variation of the asymmetric line profiles quite well. The existence of prograde $m=1$ modes close to the central star has also been directly detected by spectrally resolved interferometry of the Be stars $\zeta$ Tau (Vakili et al. 1998) and $\gamma$ Cas (Berio et al. 1999).

Okazaki (1997) argued that, although the observed density patterns appear indeed to revolve in the prograde sense, the proposed mechanism by Papaloizou et al. (1992) does not work for the hot early type Be star systems and he introduced a radiative force with the aim of confining the $m=1$ density waves to the inner part of these hot discs and induce prograde motions. Okazaki is indeed correct that in calculations with a rigid inner disc boundary (Savonije \& Heemskerk 1993) unrealistically low disc temperatures had to be adopted. In a more recent study of $m=1$ modes Fiřt \& Harmanec (2006) note sensitivity of model predictions to disc model parameters, but comment that the predictive power of the model is better for the cooler Be stars where radiative forces are not added. In the latter work power law disc density profiles and rigid boundaries were also assumed.

However, in this paper we show, using both semi-analytic and numerical approaches, that if there is a narrow low density region or gap between the star and its circumstellar disc, 
so that the disc no longer has a rigid inner boundary but one that is essentially freely moveable, and there is also a significant single surface density maximum not far away, prograde $m=1$ modes with periods of a few years can be obtained even for hot Be star systems. Although the mechanism by which a Be star ejects matter into the circumstellar disc is poorly understood, we note that for the mechanisms suggested in recent studies, such as non-radial oscillations in the rapidly rotating Be star (Rivinius et al. 2001; Savonije 2005), or magnetic effects, one may indeed expect such a gap region to form when material with specific angular momentum larger than that corresponding to equatorial break up rotation is impulsively ejected from the surface during an outburst.

The plan of the paper is as follows: in Sect. 2 we give the basic equations for the oscillation modes and consider them in the low frequency limit. In that limit we use variational principles to show that rigid wall conditions are the least favourable for prograde modes and derive conditions on the stellar apsidal motion constant for their existence. These are found explicitly for a class of surface density profiles, with inner narrow low surface density regions lying interior to nearby global surface density maxima that are also considered numerically. The semi-analytic and numerical approaches were found to be consistent.

In Sect. 3 we go on to apply the model to a hot Be star: $\gamma$ Cas (B0.5 IVe) and a cool Be star: $88 \mathrm{Her}$ (B7 Ve). In both these cases prograde modes with reasonable periods are found for an appropriate degree of rotatonal distortion and apsidal motion constant, without the need to introduce an extra radiation force. Finally in Sect. 4, we summarize our conclusions.

\section{Basic equations for $\boldsymbol{m}=1$ density waves}

Let us introduce a non rotating cylindrical coordinate system $(r, \varphi, z)$ with its centre coinciding with that of the Be star. We assume the circumstellar discs about Be stars to be geometrically thin $(z \ll r)$, near Keplerian and also assume hydrostatic equilibrium in the $z$-direction $\left(v_{z}=0\right)$. For simplicity we work with a two dimensional approximation by integrating over the $z$-direction. The $z$-averaged equations of motion and the equation of continuity follow as

$$
\begin{aligned}
& \frac{\partial v_{r}}{\partial t}+v_{r} \frac{\partial v_{r}}{\partial r}+\frac{v_{\varphi}}{r} \frac{\partial v_{r}}{\partial \varphi}-\frac{v_{\varphi}^{2}}{r}=-\frac{1}{\Sigma} \frac{\partial \Pi}{\partial r}-\frac{\partial \Psi}{\partial r} \\
& \frac{\partial v_{\varphi}}{\partial t}+v_{r} \frac{\partial v_{\varphi}}{\partial r}+\frac{v_{\varphi}}{r} \frac{\partial v_{\varphi}}{\partial \varphi}+\frac{v_{r} v_{\varphi}}{r}=-\frac{1}{\Sigma r} \frac{\partial \Pi}{\partial \varphi}-\frac{1}{r} \frac{\partial \Psi}{\partial \varphi} \\
& \frac{\partial \Sigma}{\partial t}+\frac{1}{r} \frac{\partial\left(\Sigma r v_{r}\right)}{\partial r}+\frac{1}{r} \frac{\partial\left(\Sigma v_{\varphi}\right)}{\partial \varphi}=0
\end{aligned}
$$

where we have introduced a vertically integrated surface density and pressure

$\Sigma=\int_{-\infty}^{\infty} \rho \mathrm{d} z$ and $\Pi=\int_{-\infty}^{\infty} P \mathrm{~d} z$.

We adopt a polytropic (isothermal) equation of state $\Pi=K \Sigma^{\gamma}$ with $\gamma=1$ and define a (constant) sound speed $c=\sqrt{\mathrm{d} \Pi / \mathrm{d} \Sigma}=$ $\sqrt{K}$ in the disc.

Since the circumstellar discs in Be star systems have negligible mass, the gravitational potential $\Psi$ is that of the central Be star. It is essential to take the rotational deformation of the rapidly rotating Be star into account. Assuming uniform rotation and only weak distortions a multipole expansion for $\Psi$ may be limited to the monopole and quadrupole terms (Papaloizou et al. 1992):

$\Psi(r)=-\frac{G M_{\mathrm{s}}}{r}-\frac{k_{2} \Omega_{\mathrm{s}}^{2} R_{\mathrm{s}}^{5}}{3 r^{3}}$

where $M_{\mathrm{s}}, R_{\mathrm{s}}$ and $\Omega_{\mathrm{s}}$ are the mass, radius and angular rotation speed of the Be star and $k_{2}$ its apsidal motion constant (Schwarzschild 1958).

The unperturbed disc is assumed axisymmetric with negligible velocity in the radial direction, i.e. with velocity $v(r)=(0, r \Omega(r))$, whereby the angular velocity $\Omega(r)$ in the disc is given by the condition for radial equilibrium

$r \Omega^{2}=\frac{G M_{\mathrm{s}}}{r^{2}}\left[1+k_{2} f^{2}\left(\frac{R_{\mathrm{s}}}{r}\right)^{2}\right]+\frac{1}{\Sigma} \frac{\mathrm{d} \Pi}{\mathrm{d} r}$

where we have used expansion (5) for the gravitational field and $f=\Omega_{\mathrm{s}} / \Omega_{\mathrm{c}}$ with $\Omega_{\mathrm{c}}^{2}=G M_{\mathrm{s}} / R_{\mathrm{s}}^{3}$ as a measure for the stellar rotation speed.

\subsection{The linearised equations for the density waves}

After substituting the Eulerian perturbations with harmonic time dependence

$\hat{\Sigma}^{\prime}(r, \varphi, t)=\Sigma^{\prime}(r) \mathrm{e}^{\mathrm{i}(\sigma t-m \varphi)}$,

$\hat{v}_{r}^{\prime}(r, \varphi, t)=v_{r}^{\prime}(r) \mathrm{e}^{\mathrm{i}(\sigma t-m \varphi)} ; \hat{v}_{\varphi}^{\prime}(r, \varphi, t)=v_{\varphi}^{\prime}(r) \mathrm{e}^{\mathrm{i}(\sigma \mathrm{t}-\mathrm{m} \varphi)}$,

which describe a density wave with $m$-fold azimuthal symmetry, in Eqs. (1)-(3) and neglecting all orders higher than linear we find, after applying the operator relations $\frac{\partial}{\partial t} \rightarrow \mathrm{i} \sigma$ and $\frac{\partial}{\partial \varphi} \rightarrow$ -(i $m)$, the linearized oscillation equations

$\frac{\mathrm{d} W}{\mathrm{~d} r}=-\mathrm{i} \frac{\left(\bar{\sigma}^{2}-\kappa^{2}\right)}{\bar{\sigma}} v_{r}^{\prime}+\frac{2 m \Omega}{\bar{\sigma} r} W$

$\frac{\mathrm{d} v_{r}^{\prime}}{\mathrm{d} r}=-\left(\frac{1}{r}+\frac{1}{\Sigma} \frac{\mathrm{d} \Sigma}{\mathrm{d} r}+\frac{m}{\bar{\sigma} r^{2}} \frac{\mathrm{d} h}{\mathrm{~d} r}\right) v_{r}^{\prime}+\mathrm{i}\left(\frac{m^{2}}{\bar{\sigma} r^{2}}-\frac{\bar{\sigma}}{c^{2}}\right) W$

where we have eliminated $v_{\varphi}^{\prime}$ and introduced

$W(r)=c^{2} \Sigma^{\prime} / \Sigma$ and $\bar{\sigma}=\sigma-m \Omega(r)$

the oscillation frequency in a locally corotating frame. The square of the epicyclic frequency is $\kappa^{2}=(2 \Omega / r)(\mathrm{d} h / \mathrm{d} r)$, with $h=r^{2} \Omega$. The angular rate at which an orbit would precess in the absence of a perturbation to the pressure force is given by

$\omega_{\mathrm{p}}=\Omega-\kappa$.

From now on we adopt $m=1$ to obtain one-armed density waves.

\subsection{Low frequency limit}

Although Eqs. (8), (9) can be readily solved numerically as they stand, we remark that the $m=1$ modes we consider are low frequency modes satisfying $|\sigma| \ll \Omega$. This situation arises because the modes correspond to elliptical distortions that precess on a long time scale compared to the orbital period. In the low frequency limit the equations may be reduced to a simplified form as in Papaloizou (2002) and Papaloizou (2005). This enables general deductions concerning the periods of oscillation and the way they are affected by boundary conditions to be made. To adapt Eqs. (8), (9) to this limit we assume that the system is of 
finite extent and such that it can be characterised by a single dynamical time scale $\Omega_{0}^{-1}$ that is everywhere significantly shorter than the precession time scale $\omega_{\mathrm{p}}^{-1}$ and such that we can write $\Omega=O\left(\Omega_{0}\right)$. We set $\omega_{\mathrm{p}}=\epsilon \omega_{\mathrm{pr}}$, where $\epsilon$ is a small parameter and $\omega_{\text {pr }}$ is a relative precession frequency scaled to be $O\left(\Omega_{0}\right)$. We define a new eigenvalue $\lambda$ also of order $\Omega_{0}$ by setting $\sigma=\epsilon \lambda$. In addition we set $W=\epsilon w$ and under the assumption that the disc is thin $c^{2}=\epsilon c_{0}^{2}$.

Equations (8) and (9) then give to lowest order in $\epsilon$

$$
\begin{aligned}
& \frac{\mathrm{d} w}{\mathrm{~d} r}=-2 \mathrm{i}\left(\lambda-\omega_{\mathrm{pr}}\right) v_{r}^{\prime}-\frac{2 w}{r} \\
& \frac{\mathrm{d} v_{r}^{\prime}}{\mathrm{d} r}=-\left(\frac{1}{2 r}+\frac{1}{\Sigma} \frac{\mathrm{d} \Sigma}{\mathrm{d} r}\right) v_{r}^{\prime}+\mathrm{i} \frac{w \Omega}{c_{0}^{2}} .
\end{aligned}
$$

Here we have used the fact that to within a correction of order $\epsilon$, we have $h=r^{2} \Omega=\sqrt{G M_{\mathrm{s}} r}$. We can eliminate $w$ from Eqs. (12) and (13) to obtain a single governing second order differential equation for $Q=\Sigma r^{1 / 2} v_{r}^{\prime}$ in the form

$\frac{\mathrm{d}}{\mathrm{d} r}\left(\frac{r^{3 / 2} c_{0}^{2}}{\Sigma \Omega}\left(\frac{\mathrm{d} Q}{\mathrm{~d} r}\right)\right)=\frac{2 r^{3 / 2}\left(\lambda-\omega_{\mathrm{pr}}\right) Q}{\Sigma}$

or in terms of the original unscaled coordinates

$\frac{\mathrm{d}}{\mathrm{d} r}\left(\frac{r^{3 / 2} c^{2}}{\Sigma \Omega}\left(\frac{\mathrm{d} Q}{\mathrm{~d} r}\right)\right)=\frac{2 r^{3 / 2}\left(\sigma-\omega_{\mathrm{p}}\right) Q}{\Sigma}$.

Equation (15) and its associated eigenvalue problem is of SturmLiouville form. This enables us to make use of the associated variational principle for the eigenfunctions and eigenvalues (Courant \& Hilbert 1953). We comment that it is possible to formulate this principle using the product of $Q$ and an arbitrary function of $r$ as perturbation variable rather than $Q$. However, the results will be the same.

\subsection{Variational principle}

In order to formulate a variational principle for the eigenvalues, we must first consider the boundary conditions. We consider the disc to have an inner boundary at $r=r_{0}$ and an outer boundary at $r=r_{1}$. We also suppose that the surface density is small but non zero at the boundaries as necessitated by our adoption of an isothermal equation of state. Then we may consider general boundary conditions for (15) of the form

$\frac{\mathrm{d} Q}{\mathrm{~d} r}=A Q$

At $r=r_{0}$ we take $A=A_{0}$ and at $r=r_{1}$ we take $A=A_{1}$. At a rigid boundary, $Q=0$, which can be thought of as corresponding to $A \rightarrow \infty$ with $\mathrm{d} Q / \mathrm{d} r$ remaining finite. The boundary condition that the Lagrangian variation of the pressure vanishes, as used in the numerical work below, corresponds to $A=(1 / \Sigma)(\mathrm{d} \Sigma / \mathrm{d} r)$.

Recalling that for Sturm-Liouville problems the eigenvalues are real and the eigenfunctions may be assumed to be real without loss of generality (Courant \& Hilbert 1953), we derive an integral expression for the eigenvalue $\sigma$ from Eq. (15) by multiplying it by $Q$ and integrating over $\left(r_{0}, r_{1}\right)$ in the form

$\sigma=\frac{I+\left[\frac{r^{3 / 2} c^{2} A}{2 \Sigma \Omega} Q^{2}\right]_{1}-\left[\frac{r^{3 / 2} c^{2} A}{2 \Sigma \Omega} Q^{2}\right]_{0}}{\int_{r_{0}}^{r_{1}} \frac{r^{3 / 2} Q^{2}}{\Sigma} \mathrm{d} r}$, where

$I=\int_{r_{0}}^{r_{1}}\left(\frac{r^{3 / 2} \omega_{\mathrm{p}} Q^{2}}{\Sigma}-\frac{r^{3 / 2} c^{2}}{2 \Sigma \Omega}\left(\frac{\mathrm{d} Q}{\mathrm{~d} r}\right)^{2}\right) \mathrm{d} r$

and the subscript $0 / 1$ denotes evaluation at the inner/outer boundary respectively. Note that when the eigenfunction decays rapidly with distance the outer boundary terms can become negligible and make no effective contribution. This is in fact the case for the eigenfunctions we later consider. However, for completeness we shall retain these terms in the general analysis.

We also recall from Eqs. (6) and (11) that correct to first order in $\epsilon$,

$\omega_{\mathrm{p}}=k_{2} \Omega f^{2}\left(\frac{R_{\mathrm{s}}}{r}\right)^{2}-\frac{c^{2}}{2 \Omega r^{2}} \frac{\mathrm{d}}{\mathrm{d} r}\left(\frac{r^{2}}{\Sigma} \frac{\mathrm{d} \Sigma}{\mathrm{d} r}\right) \equiv k_{2} \omega_{\mathrm{p}, \mathrm{e}}+\omega_{\mathrm{p}, \mathrm{c}}$

and to adequate accuracy $\Omega$ may be replaced by the Keplerian value $\Omega_{\mathrm{K}}=\left(G M_{\mathrm{s}} / r^{3}\right)^{1 / 2}$ in the above.

The expression (17) forms the basis of the variational principle for eigenvalues $\sigma$. In particular, for finite $A_{0}$ and $A_{1}$, the largest eigenvalue, associated with an eigenmode with no zeros, is an absolute maximum with respect to variation with respect to arbitrary functions $Q$. When a rigid wall condition is applied at a boundary, the same variational formalism applies, but trial functions nust be restricted to those that vanish at that boundary.

In this paper we are concerned with the question of the existence of prograde modes which have $\sigma>0$. This issue is seen to be equivalent to that of whether the maximum of (17) is positive with respect to evaluation with all permissible trial functions.

\subsection{The existence of prograde modes and the dependence on boundary conditions}

We here point out that the existence of prograde modes is boundary condition sensitive by firstly pointing out that there exists sets of boundary conditions for which the existence of prograde modes is assured. We go on to prove that a rigid wall boundary condition applied at either boundary minimizes the maximum of (17) and thus is the worst form of boundary condition for enabling prograde modes.

Consider the equation derived from (15) when $k_{2}=0$ and $\sigma=0$. This is, with the help of (19), found to be

$\frac{\mathrm{d}}{\mathrm{d} r}\left(\frac{r^{3}}{\Sigma}\left(\frac{\mathrm{d} Q}{\mathrm{~d} r}\right)\right)=\frac{Q r}{\Sigma} \frac{\mathrm{d}}{\mathrm{d} r}\left(\frac{r^{2}}{\Sigma} \frac{\mathrm{d} \Sigma}{\mathrm{d} r}\right)$.

In principle a solution of (20), which we denote by $Q=Q_{0}$, can be used to define values of $A_{0}$ and $A_{1}$ to use for the boundary conditions. In fact $A_{1}$ can be specified and then $A_{0}$ determined by inward integration from the outer boundary. We comment that a specific example of a solution for a power law $\Sigma \propto r^{-\gamma}$ is $Q_{0} \propto$ $r^{-\delta}$, where $\delta=1+\gamma / 2+\sqrt{1+\gamma^{2} / 4}$.

The boundary conditions, or equivalently values of $A_{0}$ and $A_{1}$ so determined, are such that when the general eigenvalue problem is solved, there will be a prograde mode with $\sigma>0$. This follows immediately from the fact that if the trial function $Q=Q_{0}$ is adopted, only the term proportional to $k_{2}$ survives in (17) which must then be positive. Hence the maximum eigenvalue $\sigma$ must also be positive.

The fact that a rigid wall boundary condition always reduces a maximum of (17) follows from the fact that adopting a rigid wall boundary condition through restricting trial functions to be such that $Q=0$ at one of the boundaries is equivalent to restricting the search for a maximum to a subset of the possible trial 
functions that are available when a rigid wall condition is not used. In the latter case $Q$ is unrestricted at the boundary. Thus, other things being equal, changing from a non rigid wall condition to a rigid wall condition can only reduce the maximum eigenvalue $\sigma$.

\subsection{A condition on $k_{2}$ for the existence of a prograde mode}

From the variational principle it follows that it is sufficient for a prograde mode to exist that the numerator of (17) be positive for some $Q$ which leads to

$$
\begin{aligned}
& \int_{r_{0}}^{r_{1}}\left(\frac{r^{3 / 2} \omega_{\mathrm{p}} Q^{2}}{\Sigma}\right.\left.-\frac{r^{3 / 2} c^{2}}{2 \Sigma \Omega}\left(\frac{\mathrm{d} Q}{\mathrm{~d} r}\right)^{2}\right) \mathrm{d} r \\
&+\left[\frac{r^{3 / 2} c^{2} A}{2 \Sigma \Omega} Q^{2}\right]_{1}-\left[\frac{r^{3 / 2} c^{2} A}{2 \Sigma \Omega} Q^{2}\right]_{0}>0 .
\end{aligned}
$$

Using (19) this leads to

$$
\begin{aligned}
k_{2} \int_{r_{0}}^{r_{1}} \frac{r^{3 / 2} \omega_{\mathrm{p}, \mathrm{e}} Q^{2}}{\Sigma} \mathrm{d} r \geq & \int_{r_{0}}^{r_{1}} \frac{r^{3 / 2}}{\Sigma}\left(\frac{c^{2}}{2 \Omega}\left(\frac{\mathrm{d} Q}{\mathrm{~d} r}\right)^{2}-\omega_{\mathrm{p}, \mathrm{c}} Q^{2}\right) \mathrm{d} r \\
& -\left[\frac{r^{3 / 2} c^{2} A}{2 \Sigma \Omega} Q^{2}\right]_{1}+\left[\frac{r^{3 / 2} c^{2} A}{2 \Sigma \Omega} Q^{2}\right]_{0} .
\end{aligned}
$$

This in turn leads to a variational principle for the minimum value of $k_{2}$ for which a prograde mode exists. This is just the minimum that can be found from (22) for any permissible $Q$. Again because the possible trial functions for rigid wall conditions are a subset of those allowed for other boundary conditions, the minimum is larger in that case as is the corresponding value of $k_{2}$. Accordingly free boundary conditions are always more favourable for prograde modes than are rigid wall conditions.

From general scaling arguments, we would expect that for surface densities sufficiently peaked near $r=R_{\mathrm{s}}$, that this leads to a sufficient condition of the form

$k_{2} f^{2}>\frac{\zeta c^{2} R_{\mathrm{s}}}{G M_{\mathrm{s}}}$

where $\zeta$ is a constant depending on the details of the surface density profile and boundary conditions but which is larger for rigid wall conditions relative to for example free boundary conditions. A condition of the same form as (23) was found in the case of a simple example presented below.

The variational principle for the minimum $k_{2}$ for which prograde modes are possible is equivalent to the eigenvalue problem obtained from Eq. (15) when $\sigma$ is set to zero and $k_{2}$ is taken to be the eigenvalue. The minimum eigenvalue gives the minimum value of $k_{2}$ for which prograde modes occur.

\subsection{Density profiles that are highly peaked near the inner edge}

We briefly consider the application of (22) to systems with surface densities that are highly peaked near the inner edge. To proceed we note that the trial function $Q=\Sigma$ satisfies the free boundary condition (16). Using this and adopting a surface density that is such that it and its radial derivative are very small at the boundaries so that the boundary terms become negligible, after some integrations by parts, (22) gives that it is sufficient for prograde modes to exist that

$k_{2} \int_{r_{0}}^{r_{1}} \Sigma r^{3 / 2} \Omega f^{2}\left(\frac{R_{\mathrm{s}}}{r}\right)^{2} \mathrm{~d} r \geq \int_{r_{0}}^{r_{1}} \frac{\Sigma c^{2}}{\Omega r^{1 / 2}} \mathrm{~d} r$, or after using Kepler's law

$\int_{r_{0}}^{r_{1}} \Sigma r^{3 / 2} \Omega\left(k_{2} f^{2} \frac{R_{\mathrm{s}}^{2}}{r^{2}}-\frac{c^{2} r}{G M}\right) \mathrm{d} r \geq 0$.

From the above it is apparent that if $\Sigma$ is highly peaked, with a maximum at $r=R_{\max }$ say, that prograde modes are guaranteed if $k_{2} f^{2}>c^{2} R_{\max }^{3} /\left(G M R_{\mathrm{s}}^{2}\right)$. Thus for $R_{\max }=1.6 R_{\mathrm{s}}$, we obtain prograde modes if $k_{2} f^{2}>4.1\left(c^{2} R_{\mathrm{s}}\right) /(G M)$. We now consider a specific example of a family of profiles and evaluate the condition exactly. This turns out to be of similar form. Thus, as has also been confirmed by normal mode calculations, the general form of our results does not depend on details of the surface density profile.

\subsection{A simple example}

We consider a surface density profile with a form very similar to that adopted below for our numerical work, being given by

$\Sigma(r)=\mathcal{A}\left(\frac{R_{\mathrm{s}}}{r}\right)^{\alpha} \exp \left(\frac{-\beta R_{\mathrm{s}}^{3}}{r^{3}}\right)$,

where $\mathcal{A}, \alpha$, and $\beta$ are constants.

For this form of surface density, the equation for $Q$, when $\sigma=0$ is

$\frac{\mathrm{d}^{2} Q}{\mathrm{~d} x^{2}}+\frac{\mathrm{d} Q}{\mathrm{~d} x}\left(\frac{3+\alpha}{x}-\frac{3 \beta}{x^{4}}\right)=Q\left(\frac{-\alpha}{x^{2}}-\frac{2 \mathcal{K}_{2}}{x^{5}}\right)$.

Here $x=r / R_{\mathrm{S}}$ and $\mathcal{K}_{2}=k_{2} f^{2} G M_{\mathrm{s}} /\left(c^{2} R_{\mathrm{s}}\right)+3 \beta$. In terms of $z=x^{-3}$, we find

$9 z^{2} \frac{\mathrm{d}^{2} Q}{\mathrm{~d} z^{2}}+\frac{\mathrm{d} Q}{\mathrm{~d} z}\left(3(1-\alpha) z+9 \beta z^{2}\right)=Q\left(-\alpha-2 \mathcal{K}_{2} z\right)$.

Solutions of (28) can be found as power series expansions. We adopt the solution that vanishes most rapidly as $z \rightarrow 0$, or equivalently $x \rightarrow \infty$ which can be written, to within an arbitrary normalization factor, in terms of a confluent hypergeometric function in the form (Whittaker \& Watson 1963)

$Q=z^{q}{ }_{1} F_{1}\left(2 \mathcal{K}_{2} /(9 \beta)+q ; r / 9+1 ;-\beta z\right)$,

where $r=3 \sqrt{4+\alpha^{2}}$, and $q=(6+3 \alpha+r) / 18$. Specification of an inner boundary condition, here taken at $r=R_{\mathrm{S}}$ or $z=1$, then determines $\mathcal{K}_{2}$ and hence $k_{2} f^{2}$ as an eigenvalue. The smallest eigenvalue for fixed $\alpha$ and $\beta$ then leads to the smallest value of $k_{2}$ for which prograde modes can exist. For a rigid wall condition $Q=0$ and for vanishing Lagrangian change in pressure, $3 z \mathrm{~d} Q / \mathrm{d} z=Q(\alpha-3 \beta)$, at $z=1$.

Once an inner boundary condition is adopted, it is a simple matter to obtain the smallest value of $k_{2} f^{2} G M_{\mathrm{s}} /\left(c^{2} R_{\mathrm{s}}\right)$ for which prograde modes are possible. Results for various $\alpha$ and $\beta$ and different inner boundary conditions are given in Table 1 . These are fully consistent with the numerical work we have carried out (see below for further discussion).

\subsection{Strength of the quadrupole factor $k_{2} f$}

The structure and dynamical behaviour of the disc depends on the strength of the quadrupole term in Eq. (5) which is proportional to the factor $k_{2} f^{2}$. Unfortunately, the apsidal motion constant $k_{2}$ and the stellar rotation speed factor $f=\Omega_{\mathrm{s}} / \Omega_{\mathrm{c}}$ are poorly determined stellar parameters. For B-type main sequence stars stellar evolution calculations yield for $k_{2}$ typical values 
Table 1. The values of the quadrupole factor $k_{2} f^{2}$ above which there is a prograde $m=1$ mode in the disc determined from the semi-analytic theory as a function of the disc model parameters $\alpha$ and $\beta$ defined in (26), and the type of inner boundary condition.

\begin{tabular}{cccc}
\hline \hline Inner disc boundary & $\alpha$ & $\beta$ & $k_{2} f^{2} G M_{\mathrm{s}} /\left(c^{2} R_{\mathrm{s}}\right)$ \\
\hline Free & 2 & 7.5 & 4.7 \\
Free & 2 & 6 & 3.9 \\
Free & 2.5 & 5.5 & 3.1 \\
Free & 2.5 & 7.5 & 4.0 \\
Rigid & 2.5 & 0 & 17.3 \\
\hline
\end{tabular}

in the range $2.5 \times 10^{-3}-10^{-2}$ (Claret \& Gimenez 1991; Claret 1995). The rotation speeds are derived from line width measurements which includes the uncertain inclination factor $\sin i$ and the effect of rotational gravity darkening which is thought to have lead to too low estimates of the rotation speeds in earlier studies (Porter \& Rivinius 2003) due to underweighting of the cooler, most rapidly rotating equatorial region. Yet it generally remains unclear how close a particular Be star is to critical rotation, although in some cases interferometry may be helpful (Tycner et al. 2004) and suggest large rotational deformation, e.g. for the Be star $\alpha$ Eri (Domiciano de Souza et al. 2003). This has been questioned by Vinicius \& Levenhagen (2006), who deduce a lower ratio of equatorial to polar radius for this star and a rotation rate $\Omega / \Omega_{\mathrm{c}} \simeq 0.8$.

Cranmer (2005) made a new detailed statistical study of the threshold rotation rates for the formation of Be star discs. He comes to the conclusion that the early type Be stars (O7e-B2e) exhibit a spread of equatorial rotation rates between a lower limit around $0.4-0.5 \Omega_{\mathrm{c}}$ and an upper limit near break up speed $\Omega_{\mathrm{c}}$. He finds the spread in rotation rates to narrow progressively when considering later spectral types, approaching critical rotation speeds for the A0e stars.

Frémat et al. (2005) studied the effects of fast rotation in early type stars and calculated synthetic spectra with classical plane-parallel non-LTE model atmospheres for rapidly rotating stars. They too find that gravity darkening can lead to serious underestimates of $V \sin i$ values when considering the rotational broadening of spectral lines. By comparing with spectra of $130 \mathrm{Be}$ stars they conclude that the average angular rotation speed of Be stars is most probably $\Omega_{\mathrm{s}} \simeq 0.9 \Omega_{\mathrm{c}}$. From all this we infer that for the hot, early type Be stars the quadrupole factor $k_{2} f^{2}$ may lie somewhere between $4 \times 10^{-4}$ and $10^{-2}$, unfortunately quite a wide range.

\section{Numerical results}

\subsection{Adopted disc structure}

For the (poorly known) density distribution in the equilibrium disc we adopt the same form (26) as used in the semi-analytic analysis above, except for an additional constant $B$ which is chosen so as to provide a floor to the surface density such that it does not decrease to below $5 \times 10^{-4}$ of its maximum value (and inhibit a solution for isothermal discs):

$\Sigma(r)=\frac{\mathcal{A}}{r^{\alpha}} \exp \left(\frac{-\beta}{r^{3}}\right)+B$.

The exponential factor with the $\beta$ parameter is introduced to cut off the steeply increasing density profile near the star and to produce a gap (or low density zone) just outside the stellar surface. The formation of a gap seems a rather natural assumption for

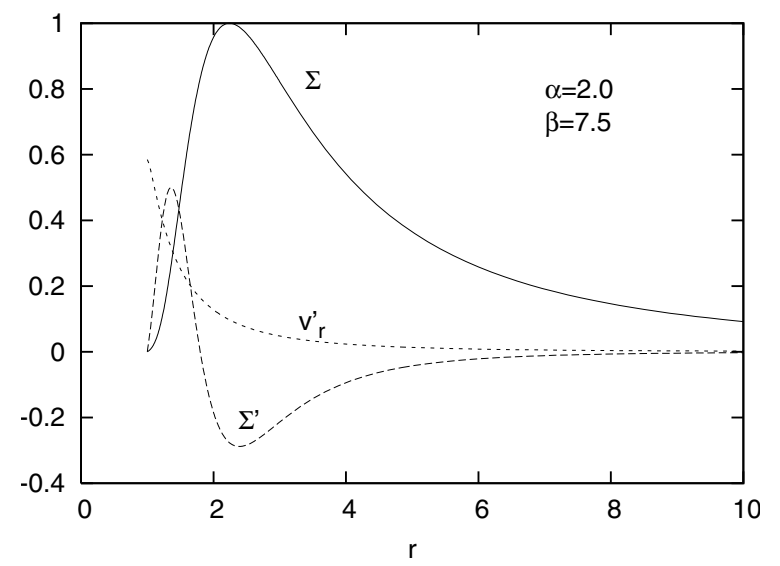

Fig. 1. A model of $\gamma$ Cas with $M_{\mathrm{s}}=15 M_{\odot}, R_{\mathrm{s}}=8 R_{\odot}$ and $T_{\mathrm{e}}=$ $25000 \mathrm{~K}$. The disc profile is defined by $\alpha=1.5$ and $\beta=7.5$ for which the disc has a free inner boundary (with a narrow gap between disc and star) as well as a free outer boundary near $r \sim 40$. Plotted are the density profile $\Sigma(r)$ of the unperturbed disc together with the eigenfunctions $\Sigma^{\prime}$ and $v_{r}^{\prime}$, in fact $\mathfrak{J}\left(v_{r}^{\prime}\right)$, for the $m=1$ mode with $P \simeq 7 \mathrm{yr}$. We only show the inner 10 stellar radii from the stellar centre. The vertical scale is arbitrary.

circumstellar discs that are generated by impulsive mass ejection from the central star. It allows us to apply a free boundary condition at the inner disc edge. As indicated above, the eigenvalue solution is only sensitive to the integrated disc properties and independent of the precise shape of the density maximum, as long as it is close to the star. Therefore simulations with an exponential cut off for different values of $\beta$ should be adequate to probe the dependence of the results to the disc's density profile near the star.

From here on we work in units for which $G=M_{\mathrm{S}}=R_{\mathrm{S}}=1$ and, as for the semi-analytic discussion, take $r=1$ for the inner disc boundary $r_{0}$, neglecting the small width of the gap between star and disc (the stellar radii being not well known anyhow). Observationally derived (crude) power laws $\left(r^{-n}\right)$ for the density distribution $\rho(r)$ in the circumstellar discs are typically $n \sim 2-4$ (Waters et al. 1987). Adopting $n=3$ or 4 we find for a semiKeplerian disc with disc semi-thickness (in $z$ direction) $H \sim c / \Omega$ that $\alpha \simeq 3 / 2$ or $5 / 2$.

The profile of the surface density $\Sigma(r)$ for $\alpha=2$ and $\beta=7.5$ is plotted in Fig. 1. This disc extends quite far out from the star to $r \simeq 40$. The deviations from Keplerian rotation are strongest near the inner disc boundary where in our units $\Omega=1.0103$ (1.000) and the epicyclic frequency $\kappa=0.9881$. At $r=10$ the deviation from Keplerian rotation is already small with $\Omega=0.0308$ $(0.0316)$ and $\kappa=0.0307$. The numbers quoted in brackets refer to the angular speed in a purely Keplerian disc (for which $\Omega=\kappa)$.

We study the influence of the disc structure on the obtained oscillation periods by also considering more compact discs with slightly different values of $\alpha$ and $\beta$. We assume the temperature in the isothermal discs is given by $T_{\mathrm{d}}=2 / 3 T_{\mathrm{e}}$, where $T_{\mathrm{e}}$ is the Be star's effective temperature, unless stated otherwise.

\subsection{Boundary conditions and numerical solution}

When doing numerical work we do not make a low frequency approximation as above but solve Eqs. (8), (9) directly. However, as above, and unlike for previous calculations of $m=1$ modes in circumstellar discs (Savonije \& Heemskerk 1993; Okazaki 1997) we consider the existence of a small low surface density 
region, or gap, between the star and surrounding disc. Formation of a gap may be expected when the disc material was ejected from the star as a result of non-radial oscillations (Rivinius et al. 2001) or magnetic levitation with specific angular momentum larger than $\Omega_{\mathrm{s}} R_{\mathrm{s}}^{2}$.

We apply free boundary conditions to both the inner and outer boundary of the disc and require the Lagrangian pressure perturbation $\delta \Pi$ to vanish at both boundaries, or

$v_{r}^{\prime}=\frac{\mathrm{i} \bar{\sigma}}{c^{2}} \frac{W}{\frac{\mathrm{d} \ln \Sigma}{\mathrm{d} r}}$

unless specifically noted otherwise. These conditions correspond to those used in the semi-analytic discussion. We integrate Eqs. (8), (9) using a fourth order Runge-Kutta method with adaptive stepsize (Press et al. 1986) away from both disc boundaries, adopting an arbitrary boundary value $W=1$, to a conveniently chosen fitting point in the central region of the disc. We then search for that (real) oscillation frequency $\sigma$ for which the difference in the ratio of $W / v_{r}^{\prime}$ determined from integrations starting on different sides of the fitting point becomes sufficiently small. The linear solution can then be scaled on each side so that $W$ and $v_{r}^{\prime}$ match separately. We comment that for the cases considered below, the location of the outer boundary was chosen such that further extension did not affect the results.

\subsection{A hot Be star: $\gamma$ Cas (B0.5 IVe)}

We now consider models for $\gamma$ Cas, the brightest Be star in the northern sky for which we adopt $M_{\mathrm{S}}=15 M_{\odot}$, radius $R_{\mathrm{S}}=8 R_{\odot}$ and effective temperature $T_{\mathrm{e}}=25000 \mathrm{~K}$. Fiřt \& Harmanec (2006) adopted the same radius, but a larger mass $\left(18.3 M_{\odot}\right)$ and higher effective temperature $\left(T_{\mathrm{e}}=33000 \mathrm{~K}\right)$ for this star. We note that, according to the semi-analytic result in Sect. 2.5, these larger values require the quadrupole term $k_{2} f^{2}$ to be larger by only a factor $\propto c^{2} / M_{\mathrm{s}}$, i.e. by a factor $\simeq 1.08$ in order to obtain the same oscillation period. The observed V/R quasi-periods of $\gamma$ Cas fall in the range 4-7 years (Doazan et al. 1987).

We first consider the quite arbitrary semi-Keplerian circumstellar disc model described by (30) with $\alpha=2$ and $\beta=7.5$, for which the density profile $\Sigma(r)$ in the inner disc is shown in Fig. 1. Note that if we adopt a rigid inner boundary to the disc (i.e. adopt $\beta=0$, so that there is no gap) with $v_{r}^{\prime}=0$ there cannot exist prograde $m=1$ modes in the hot disc model considered here as the pressure forces dominate over the gravitational quadrupole effect and we obtain retrograde solutions that propagate all over the disc (Papaloizou et al. 1992).

However, if we assume there is a (narrow) low-density region or gap between the star and the disc and consider a free inner disc boundary with a non-vanishing radial velocity of the gas, the situation changes drastically. Then the gas in the disc can oscillate in the radial direction right from the inner boundary where the gravitational quadrupole term is strongest, so that the latter term can dominate the dynamic behaviour. The numerical calculations show that a free inner boundary leads to a very different outcome whereby we do find prograde $m=1$ oscillations in the observed period range, see Fig. 2, consistent with our semi-analytic estimates. An observed V/R period of 7 years would for the disc model with $\alpha=2$ and $\beta=7.5$ thus require a rotation parameter $k_{2} f^{2}=0.0065$ which is rather large, although in the allowed range, see Sect. 2.8. Figure 1 shows the shape of the eigenfunctions for a $m=1$ mode with period $P=6.6$ years. The shape of the eigenfunctions varies little with $k_{2} f^{2}$ (or period). The shape of the density perturbation with a peak near

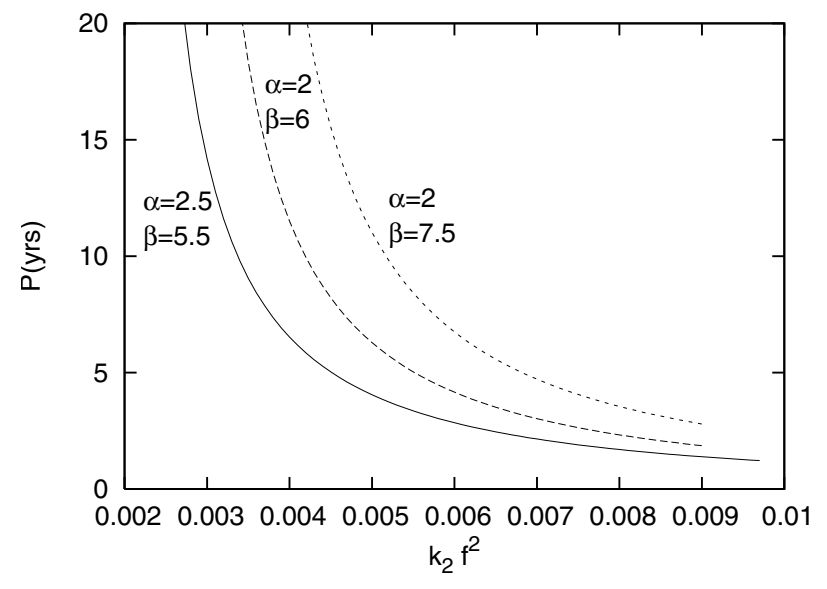

Fig. 2. The oscillation (or $\mathrm{V} / \mathrm{R}$ ) periods in years versus the quadrupole factor $k_{2} f^{2}$ of (prograde) $m=1$ modes for the extended disc in the $\gamma$ Cas model with $\alpha=2$ and $\beta=7.5$ shown in Fig. 1, together with the results for the compact disc with $\alpha=2.5$ and $\beta=5.5$ shown in Fig. 3 . The observed V/R quasi-periods for $\gamma$ Cas range from 4-7 years. To see the effect of a steeper decline of $\Sigma$ towards the inner disc boundary, we have also plotted the results for $\beta=6$.

$r=2.3$ is in fact rather similar to what Berio et al. (1999) have inferred from their interferometric observations of $\gamma$ Cas: they deduce a prograde revolving $m=1$ pattern centred near $r \sim 2.5$ from the stellar centre.

For the extended disc shown in Fig. 1 the also observed V/R period of 4 years would require a value of the quadrupole factor $k_{2} f^{2} \sim 0.008$, closer to the upper limit of 0.01 . However, the oscillation periods of the $m=1$ modes studied here appear sensitive to the size and adopted density profile of the disc. An alternative profile $\Sigma(r)$ corresponding to $\alpha=2.5$ and $\beta=5.5$ is plotted in Fig. 3, together with the $m=1$ eigenfunctions for $k_{2} f^{2}=0.005$. The maximum for $\Sigma$ has shifted slightly more inward compared to the profile shown in Fig. 1, while the density vanishes already near $r \sim 9$. Note that in this case the disc extends to only $r \simeq 9$, i.e. just beyond the propagation boundary of the $m=1$ mode near $r \simeq 6.5$. For this more compact (measured by the location of the surface density maximum) disc the oscillation periods for a given quadrupole factor $k_{2} f^{2}$ are significantly shorter and extend to smaller rotation values, as can be seen in Fig. 2. For a compact (unevolved?) disc like this an $m=1$ oscillation period of 4 years requires a smaller quadrupole factor of $k_{2} f^{2} \simeq 0.005$. The $m=1$ oscillation periods for an intermediate disc with $\alpha=2$ and $\beta=6$ are also plotted in Fig. 2 .

One can speculate that just after mass ejection and rebuilding of the circumstellar disc it may be rather compact and exhibits V/R quasi-periods in the lower part of the observed range while after some time, when angular momentum transport has taken place in the disc, it may get smeared out leading to longer periods of the $m=1$ modes. This type of evolution would in Fig. 2 correspond to a vertical shift from the compact disc with the plotted lower branch $(\beta=5.5)$ to the extended disc with the higher branch $(\beta=7.5)$. That the two branches are not fully consistent in that they require slightly different quadrupole factors to explain the observed range in V/R periods should not surprise us in view of the arbitrariness of our disc models. It is interesting that the evolution from a 4 to 7 year $V / R$ period occurred during the period 1970-1990 which coincided indeed with the build up of the disc around $\gamma$ Cas (Doazan et al. 1987).

It is also of interest to compare these results with those obtained using the semi-analytic theory. Values of 


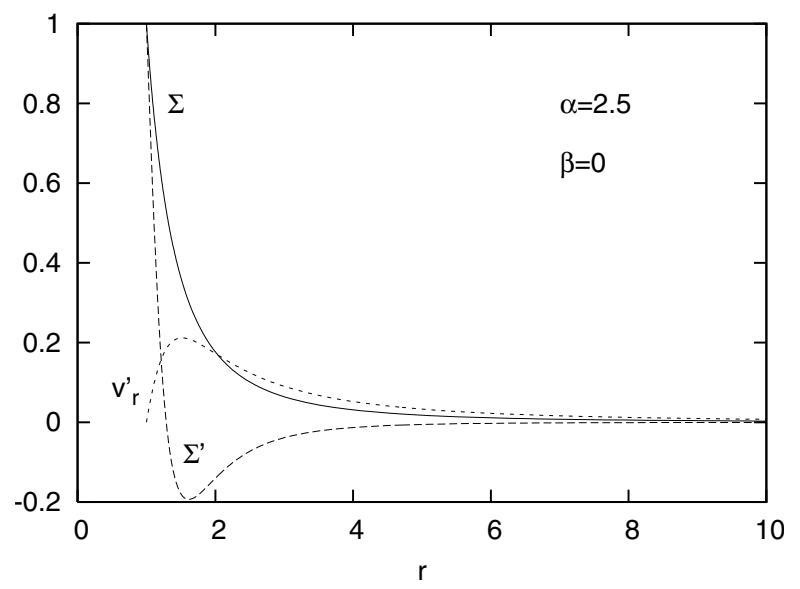

Fig. 3. A model of $\gamma$ Cas with a compact disc defined by $\alpha=2.5$ and $\beta=$ 5.5. The circumstellar disc extends only to $r \sim 9$, with a steeper decay of $\Sigma$ towards the inner boundary in comparison with the disc shown in Fig. 1. Plotted are the density profile $\Sigma(r)$ of the unperturbed disc with the eigenfunctions $\Sigma^{\prime}$ and $v_{r}^{\prime}$ for the $m=1$ mode with period $P \simeq 4 \mathrm{yr}$. The vertical scale is arbitrary.

$k_{2} f^{2} G M_{\mathrm{s}} /\left(c^{2} R_{\mathrm{s}}\right)$ above which prograde modes exist for the values of $\alpha$ and $\beta$ used in Fig. 2 are given in Table 1. These can be converted to values of $k_{2} f^{2}$ using $G M_{\mathrm{s}} /\left(R_{\mathrm{s}} c^{2}\right)=1.5 \times 10^{3}$ which is appropriate to this model. These values correspond to the asymptotic limits for $P \rightarrow \infty$ in Fig. 2. They are found to be $0.0031,0.0026$ and 0.0021 for the pairs $(\alpha=2, \beta=7.5)$, $(\alpha=2, \beta=6)$ and $(\alpha=2.5, \beta=5.5)$, respectively, and are accordingly consistent with the plots in Fig. 2 .

\subsection{A cool Be star: $88 \mathrm{Her}(B 7 \mathrm{Ve})$}

We now turn to models for $88 \mathrm{Her}$, a Be star with estimated mass of $M_{\mathrm{s}}=5 M_{\odot}$ with $R_{\mathrm{s}}=4 R_{\odot}$ and $T_{\mathrm{e}}=12500 \mathrm{~K}$. For this cooler Be star system we consider two different discs, one with $\alpha=2.5$ and $\beta=7.5$ as before, i.e. with a gap between star and disc, whereby the disc extends out to $r \sim 19$, and another model with $\beta=0$, so that the inner disc touches the stellar surface. The boundary layer that exists in that case (assuming $\Omega_{\mathrm{s}}<\Omega_{\mathrm{c}}$ ) between the star and the inner disc is ignored here and replaced by the condition of a rigid wall: $v_{r}^{\prime}=0$. The resulting disc profiles and calculated $m=1$ eigen modes are plotted in Fig. 4 and 5, respectively. The periods of the calculated prograde $m=1$ modes are shown in Fig. 6. For the disc model that touches the stellar surface $(\beta=0)$ we have calculated the $m=1$ modes for the standard disc temperature $T_{\mathrm{d}}=2 / 3 T_{\mathrm{e}}$ and also for a cooler disc with $T_{\mathrm{d}}=6000 \mathrm{~K}$. The disc model with free inner boundary $(\beta=7.5)$ exhibits prograde $m=1$ modes with much lower oscillation periods and for significantly smaller values of the quadrupole factor $k_{2} f^{2}$ as compared to the discs with a rigid inner boundary.

To compare these results with those obtained using the semianalytic theory, we use the values of $k_{2} f^{2} G M_{\mathrm{s}} /\left(c^{2} R_{\mathrm{s}}\right)$ above which prograde modes exist given in table 1 . To obtain values of $k_{2} f^{2}$ we take $G M_{\mathrm{s}} /\left(R_{\mathrm{s}} c^{2}\right)=2 \times 10^{3}$ which is appropriate to the model with $(\alpha=2.5, \beta=7.5)$ and $G M_{\mathrm{s}} /\left(R_{\mathrm{s}} c^{2}\right)=2.8 \times 10^{3}$ for the model with $(\alpha=2.5, \beta=0)$ and $T_{\mathrm{d}}=6000 \mathrm{~K}$. These values correspond to the asymptotic limits for $P \rightarrow \infty$ in Fig. 6 and are found to be 0.002 and 0.006 , respectively. These results are consistent with the data shown in Fig. 6. In particular they confirm the much larger critical value of $k_{2} f^{2}$ that applies in the case when a rigid wall inner boundary condition was applied to a distributed disc surface density profile.

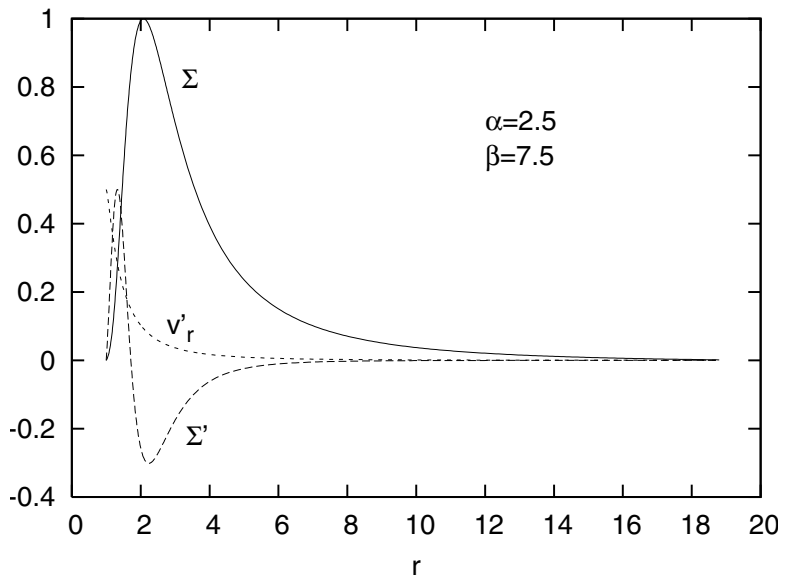

Fig. 4. A model of 88 Her with $M_{\mathrm{s}}=5 M_{\odot}, R_{\mathrm{s}}=4 R_{\odot}$ and $T_{\mathrm{e}}=$ $12500 \mathrm{~K}$ and with a disc defined by $\alpha=2.5$ and $\beta=7.5$. The disc has a free inner boundary and extends to $r \simeq 19$. Plotted are the density profile $\Sigma(r)$ of the unperturbed disc with the eigenfunctions $\Sigma^{\prime}$ and $v_{r}^{\prime}$ for the $m=1$ mode with $P \simeq 3.3 \mathrm{yr}$. The vertical scale is arbitrary.

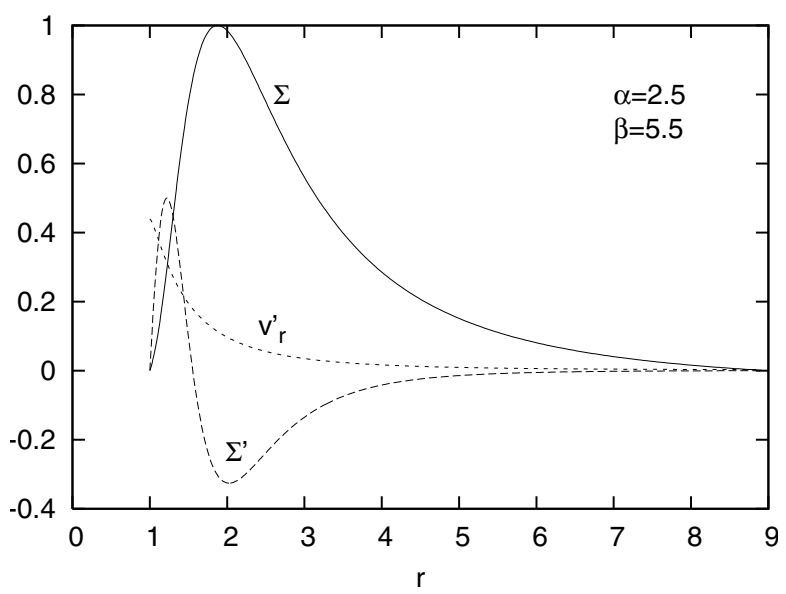

Fig. 5. A model of 88 Her with a disc defined by $\alpha=2.5$ and $\beta=0$ with $T_{\mathrm{d}}=8333 \mathrm{~K}$. The disc touches the stellar surface at $\mathrm{r}=1$ where it feels a rigid inner boundary $\left(v_{r}^{\prime}=0\right)$, the free outer disc boundary is at $r \simeq 40$. Plotted are the density profile $\Sigma(r)$ of the unperturbed disc with the eigenfunctions $\Sigma^{\prime}$ and $v_{r}^{\prime}$ for the $m=1$ mode with $P \simeq 15 \mathrm{yr}$. The vertical scale is arbitrary.

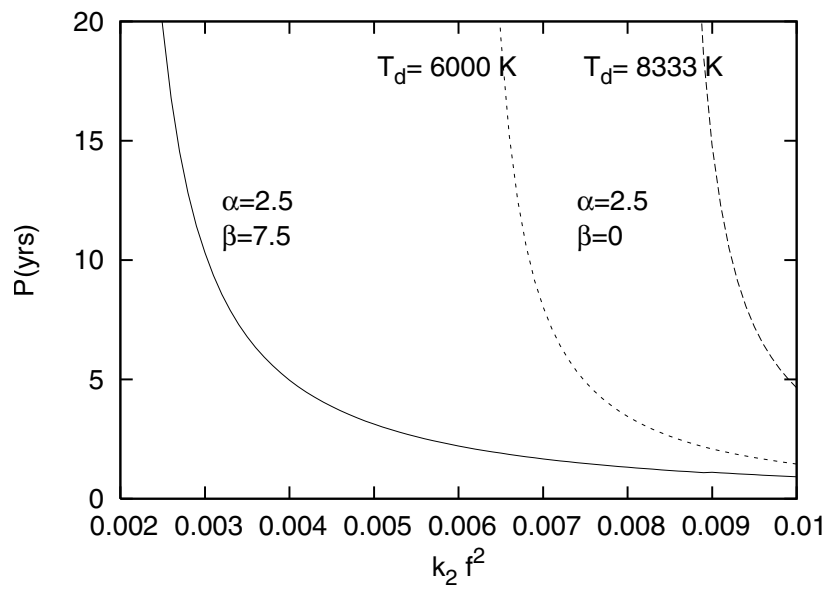

Fig. 6. The oscillation (or V/R) periods in years versus $k_{2} f^{2}$ for the calculated (prograde) $m=1$ modes for a model of $88 \mathrm{Her}$, as indicated in the figure. The results for the two disc models (for two different disc temperatures) with $\beta=0$ correspond to $m=1$ modes with rigid wall inner boundary conditions. 


\section{Conclusions}

We have investigated the effect of the rotational flattening of the rapidly spinning Be stars on low-frequency global $m=1$ oscillations in their circumstellar discs using both semi-analytic and numerical approaches.

We considered both a hot, early type Be star system like $\gamma$ Cas and a cool late type system like 88 Her and found that if it is assumed there exists a (narrow) low surface density region or gap between the star and the disc, such that the disc inner boundary is free, low-frequency global $m=1$ oscillations can be supported with periods in the observed range of the longterm $\mathrm{V} / \mathrm{R}$ variations of the Balmer emission lines for plausible values of the rotational factor $k_{2} f^{2}(\leq 0.008)$. Therefore it is not necessary to introduce radiation forces (Okazaki 1997) to obtain prograde $m=1$ modes that are contained in a oscillation cavity within a few stellar radii from a hot Be star surface.

Our calculations suggest that the more compact circumstellar discs exhibit $m=1$ modes with smaller oscillation periods than the more extended discs for which the density maximum occurs slightly further out in the disc and for which the density decreases more slowly with increasing distance from the star.

Of course in its present linear form our simple dynamical model cannot explain the full complexity of the observed variations in Be stars, in particular non-linear effects need to be included (Fiřt \& Harmanec 2006), especially in enigmatic systems like $\gamma$ Cas (Motch et al. 2005), but it appears to lead to a natural explanation for the long term $\mathrm{V} / \mathrm{R}$ variations in Be star systems, given the observational fact that there are circumstellar discs with slowly revolving $m=1$ patterns. The model naturally accounts for the observed (Berio et al. 1999; Vakili et al. 1998) confinement and the prograde character of these patterns. How the circumstellar discs are formed and how the low frequency one armed density waves therein are excited still remains a puzzle.

\section{References}

Berio, P., Stee, P., Vakili, F., et al. 1999, A\&A, 345, 203

Claret, A. 1995, A\&AS, 109, 441

Claret, A., \& Gimenez, A. 1991, A\&AS, 87, 507

Courant, R., \& Hilbert, D. 1953, Methods of Mathematical Physics, Vols. 1, 2

(New York Interscience)

Cranmer, S. R. 2005, ApJ, 634, 585

Dachs, J. 1987, in Physics of Be Stars, ed. A. Slettebak \& T. Snow (Cambridge University Press), IAU Coll., 92, 149

Doazan, V., Bourdonneau, B., Rusconi, L., Sedmak, G., \& Thomas, R. N. 1987, A\&A, 182, L25

Domiciano de Souza, A., Kervella, P., Jankov, S., et al. 2003, A\&A, 407, 47

Fiřt, R., \& Harmanec, P. 2006, A\&A, 447, 277

Frémat, Y., Zorec, J., Hubert, A.-M., \& Floquet, M. 2005, A\&A, 440, 305

Hubert, A. M. 1994, in Pulsation, Rotation and Mass Loss of Early type Stars, ed. L. Balona, H. Henrichs, \& J. Le Contel (Kluwer Academic Publisher), IAU Symp., 162, 341

Hummel, W., \& Hanuschik, R. W. 1997, A\&A, 320, 852

Hummel, W., \& Vrancken, M. 1997, A\&A, 302, 751

Motch, C., Lopes de Oliveira, R., Negueruela, I., Haberl, F., \& Janot-Pacheco, E. 2005, [arXiv: astro-ph/0512556]

Okazaki, A. T. 1991, PASJ, 43, 75

Okazaki, A. T. 1997, A\&A, 318, 548

Papaloizou, J. C. B. 2002, A\&A, 388, 615

Papaloizou, J. C. B. 2005, A\&A, 432, 757

Papaloizou, J. C. B., Savonije, G. J., \& Henrichs, H. F. 1992, A\&A, 265, L45

Porter, J. M., \& Rivinius, T. 2003, PASP, 115, 1153

Press, W. H., Flannery, B. P., Teukolsky, S., \& Vetterling, W. T. 1986, Numerical Recipes (Cambridge University Press)

Rivinius, T., Baade, D., \& Stefl, S. 2001, A\&A, 369, 1058

Savonije, G. J. 2005, A\&A, 443, 557

Savonije, G. J., \& Heemskerk, M. H. M. 1993, A\&A, 276, 409

Schwarzschild, M. 1958, Structure and Evolution of the Stars (Princeton University Press)

Telting, J. H., Heemskerk, M. H. M., Henrichs, H. F., \& Savonije, G. J. 1994, A\&A, 288, 558

Tycner, C., Hajian, A. R., Armstrong, J. T., et al. 2004, AJ, 127, 1194

Vakili, F., Mourard, D., Stee, P., et al. 1998, A\&A, 335, 261

Vinicius, M. M. F., Zorec, J. L. N., \& Levenhagen, R. 2006, A\&A, 446, 643

Waters, L. B. F., Coté, J., \& Lamers, H. J. G. 1987, A\&A, 185, 206

Whittaker, E. T., \& Watson, G. N. 1963, A course of modern analysis (Cambridge University Press) 